\title{
POLY (ADP-RIBOSYL) ATION ET RÉPARATION DE L'ADN CHEZ LES EUCARYOTES
}

\author{
Gilbert de MURCIA, Miguel MOLINETE, \\ Valérie SCHREIBER, Frédéric SMMONIN, \\ Gérard GRADWOHL, Claude NIEDERGANG, \\ Muriel MASSON et Josiane MÉNISSIER-de MURCIA
}

Institut de Biologie Moléculaire et Cellulaire

du CNRS, UPR Cancérogénèse et Mutagénèse Moléculaire et Structurale, 15 rue René Descartes, 67084 Strasbourg cedex, France

La poly(ADP-ribosyl) ation est une modification post-traductionnelle des protéines nucléaires impliquées dans le métabolisme de l'ADN. Elle constitue une réponse de la cellule aux dommages induits par des radiations ou des agents cancérigènes dans le génome. Bien que la fonction biologique précise de la poly (ADP-ribose) polymérase (PARP), enzyme qui catalyse la réaction de poly (ADP-ribosyl)ation, ne soit pas encore élucidée, un grand nombre de données expérimentales indiquent que cette activité est dépendante de la présence de coupures dans l'ADN in vivo et in vitro. L'abondance naturelle de la PARP est étroitement associée à l'état de prolifération de la cellule; la PARP est pratiquement indetectable dans des cellules hautement différenciées.

L'utilisation d'inhibiteurs compétitifs de la PARP, pour tenter de déterminer son rôle in vivo, a été controversée en raison d'effets secondaires, non spécifiques. Ces études suggèrent cependant que la PARP est nécessaire pour coordonner les évènements de réparation avec ceux de la réplication et permettre la progression de la cellule dans le cycle cellulaire.

La poly (ADP-ribose) polymérase est une enzyme modulaire, multifonctionnelle, hautement conservée dans l'évolution, présente seulement chez les eucaryotes (apparemment absente chez $S$. cerevisiae). C'est une protéine monomérique de $113.2 \mathrm{kDa}$ abondante, basique, associée à la chromatine (voir pour revues Althaus and Richter, 1987; de Murcia, et al., 1988). Son activité catalytique de base (synthèse de polyADPribose) est très fortement stimulée par la présence de coupures simple ou double brin dans l'ADN. En réponse à ces "signaux" et en présence de NAD comme substrat, la PARP catalyse la polymérisation de résidus ADP-ribose en un homopolymère branché: le poly (ADP-ribose). Ce polyanion est lié de manière covalente à des protéines nucléaires acceptrices (hétéromodification) et à la PARP elle-même (automodification). 


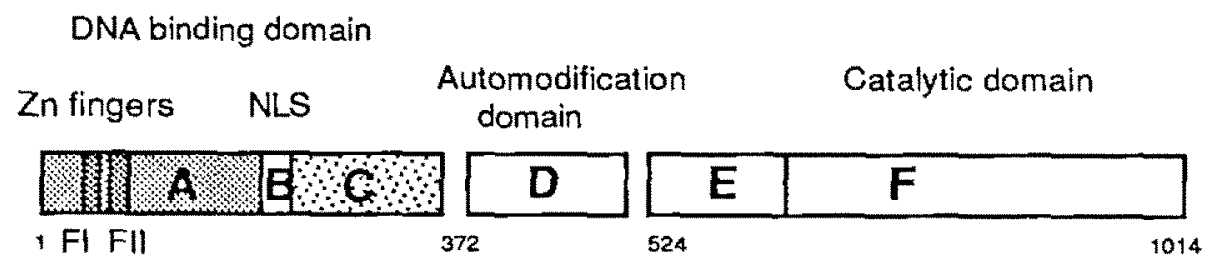

figure 1. Représentation schématique des différents domaines fonctionnels de la PARP humaine : le domaine A renferme les deux doigts à zinc (FI, FII) élêments de reconnaissance des coupures dans l'ADN; la région $B$ contient le signal de localisation nucléaire (NLS); le domaine D contient les sites d'auto polyADP-ribosylation; la région F contient le domaine catalytique.

Ces espèces modifiées sont: soit des protéines de stucture participant à l'architecture de la chromatine (histones $\mathrm{H} 1, \mathrm{H} 2 \mathrm{~B}$ ), soit des enzymes impliquées dans le métabolisme de l'ADN (topoisomérases I et II). Tous ont en commun le fait qu'ils interagissent avec l'ADN. Leur modification par poly (ADP-ribosyl) ation entraîne une forte diminution de leur affinité pour l'ADN. A partir d'un évènement affectant la continuité du génome (coupures simple brin et double brin) la PARP pourrait tout à la fois (i) moduler l'interaction protéine-ADN au voisinage du défaut, facilitant ainsi l'accès de la lésion à la machinerie de réparation par excision-resynthèse (ii) inhiber transitoirement des processus fondamentaux (transcription, replication) liés à la superhélicité de l'ADN dans le noyau en modifiant les topoisomérases. En ralentissant ainsi sa progression dans le cycle cellulaire, la cellule endommagée disposerait d'un temps suffisant pour réparer les dommages et éviterait qu'ils soient convertis en mutations transmises à la descendance. Pour une quantité massive de dommages saturant le système de réparation, la poly(ADPribosyl) ation pourrait contribuer de manière significative à l'apoptose et donc à l'élimination de cellules trop endommagées (réponse-suicide). iii) signaler à l'ensemble des autres compartiments cellulaires, le défaut détecté dans l'ADN, par la forte consommation du "pool" de NAD qu'entraîne le turn-over très rapide du poly (ADPribose) greffé aux accepteurs.

La PARP a donc potentiellement un rôle-clé dans la surveillance de l'intégrité de l'ADN chez les eucaryotes. Notre équipe s'intéresse depuis 1983 aux réactions de poly (ADP-ribosyl) ation en relation avec la structure de la chromatine et les mécanismes de réparation de l'ADN. Afin de mieux comprendre sa fonction dans ce processus, nous avons entrepris dans un premier temps, l'inventaire et l'étude des différents domaines fonctionnels présents dans l'enzyme humaine. Les résultats obtenus par notre équipe sont les suivants:

1. Le domaine $29 \mathrm{kDa} \mathrm{N}$-terminal de la PARP humaine renferme deux doigts à zinc d'un type nouveau tant sur le plan structural que fonctionnel. Il constitue l'élément de reconnaissance de coupures dans l'ADN (Gradwohl et al., 1990). 
2. La PARP contient un signal de localisation nucléaire (NLS) bipartite, capable d'assurer le transport de la PARP ou d'une protéine reporter dans le noyau (Schreiber et al., 1992)

3. Le domaine C-terminal contient le site catalytique capable de synthétiser de manière autonome du poly (ADP-ribose). Le domaine C-terminal de 40kDa possède une activité catalytique basale. Ce domaine catalytique présente de l'homologie de séquence avec les aminoacides déshydrogénases (Simonin et al., 1990,1993a, 1993b) Des cristaux du domaine catalytique $(40 \mathrm{kDa})$ ont été produits et diffractent à une résolution de $4 \AA$ pour l'instant. La connaissance de la structure tridimensionnelle de la région catalytique, pourrait déboucher sur la conception de nouveaux inhibiteurs, qui pourraient être à la base d'une chimiothérapie nouvelle visant à augmenter fortement la cytotoxicité de l'agent génotoxique utilisé (Shall, 1989).

4. Les deux propriétés caractéristiques du domaine de liaison à l'ADN (DBD) : (i) détection de coupures dans l'ADN et (ii) ciblage dans le noyau, ont été exploitées pour inhiber la PARP résidente, par surproduction du DBD dans des cellules eucaryotes. Les cellules CV-1 transfectées, exprimant de manière transitoire ce domaine, présentent le phénotype "trans-dominant négatif" : la compétition pour les coupures dans l'ADN, entre la PARP endogène et son $\mathrm{DBD}$, entraîne l'inhibition de son activité enzymatique (Küpper et al., 1990). La microinjection du DBD (surproduit dans E.coli ) dans le cytoplasme de fibroblastes humains, inhibe de la même manière l'activité PARP résidente et bloque in vivo la synthèse réparatrice d'ADN (UDS) déclenchée par le MNNG (Molinete et al. soumis pour publication). Nous avons par ailleurs, établi des lignées stables (HeLa) exprimant de façon constitutive le DBD à l'aide du vecteur épisomal pECV permettant l'expression d'un gène étranger sous le contrôle du promoteur du LTR de RSV. Les résultats préliminaires que nous avons obtenus indiquent que ces lignées sont beaucoup plus sensibles aux radiations et aux alkylants, que la lignée témoin. Leur croissance est ralentie voire arrêtée, leur survie est affectée, leur taux d'échange de chromatide-soeur (SCE) est très augmenté. Dans ces mêmes conditions, la consommation de NAD à la suite de dommages, a fortement diminué indiquant que la PARP endogène a été inhibée (Schreiber et al. en préparation). Dans les deux types d'approche, expression transitoire ou constitutive du $\mathrm{DBD}$, l'intégrité des doigts à zinc joue un rôle crucial pour que le phénotype trans-dominant négatif apparaisse.

5. En l'absence de lignée cellulaire connue, déficiente en l'activité PARP, notre objectif est maintenant d'interrompre, par recombinaison homologue, le gène codant pour cette enzyme afin d'évaluer sa fonction biologique, en créant à la fois un modèle cellulaire 
et un modèle animal. De nos jours, la disruption génique chez la souris est devenu un outil très attractif pour générer des lignées cellulaires ou des animaux d'un génotype particulier (modèles animaux pour l'étude des maladies humaines). C'est la raison pour laquelle, nous avons démarré les premiers travaux qui conduiront à l'inactivation du gène PARP chez la souris.

\section{Bibliographie}

Althaus, F.R. and Richter, C. (1987). ADP-ribosylation of proteins. Enzymology and biological significance. Mol. Biol. Biochem. Biophys. 37, 1-126.

de Murcia, G., Huletsky, A. and Poirier, G.G. (1988). Review : Modulation of chromatin structure by poly(ADP-ribosyl)ation, Biochem. Cell Biol. 66, 626-635

de Murcia, G., Ménissier-de Murcia, J. and Schreiber, V. (1991) Poly (ADP-ribose) polymerase: molecular biological aspects. BioEssays, 13, 455-462

Giner, H., Simonin, F, de Murcia, G, and Ménissier-de Murcia, J. (1992) Overproduction and large-scale purification of the human poly(ADP-ribose) polymerase using a baculovirus expression system. Gene, 114, 279-283

Gradwohl, G. , Ménissier-de Murcia, J., Molinete, M., Simonin, F. and de Murcia, G. (1989) Epression of functional zinc finger domain of human poly (ADPribose)polymerase in E. coli. Nucleic Acids Res. ,17, 7112-7112

Gradwohl, G., Ménissier-de Murcia, J., Molinete, M., Simonin, F., Koken, M., Hoeijmakers, J.H.J. and de Murcia, G. (1990). The second zinc-finger domain of poly (ADP-ribose) polymerase determines specificity for single-stranded breaks in DNA. Proc. Natl. Acad. Sci. USA 87, 2990-2994.

Küpper, J.H., de Murcia, G., and Bürkle, A. (1990) Inhibition of poly (ADPribosyl)ation by overexpressing the poly (ADP-ribose) polymerase DNA-binding domain in mammalian cells. J. Biol. Chem., 265, $18721-18724$.

Mazen, A., Ménissier-de Murcia, J., Molinete, M., Simonin, F., Gradwohl, G., Poirier, G.G. and de Murcia, G. (1989). Poly(ADP-ribose) polymerase: A novel Finger Protein. Nucleic Acids Res. 17 , 4689-4698.

Mazen, A. , Gradwohl, G. and de Murcia, G. (1988) Zinc-binding proteins detected by protein blotting. Anal. Biochem. , 172, 39-42

Ménissier-de Murcia, J., Molinete, M., Gradwohl, G., Simonin, F. et de Murcia, G. (1989). Zinc binding domain of poly(ADP-ribose) polymerase participates in the recognition of single strand breaks on DNA. J. Mol. Biol. 210, 229-233.

Schreiber, V., de Murcia, G. and Ménissier-de Murcia, J. (1992a), Le transport des protéines dans le noyau: les signaux de localisation nucléaire et leurs récepteurs.Médecine / Science , 8, 134-139.

Schreiber, V., Boeuf, H., Molinete, M., de Murcia, G. and Ménissier-de Murcia, J.(1992b) The human poly (ADP-ribose) polymerase nuclear location signal is a bipartite element functionally separate from DNA-binding and catalytic activity. EMBO J., 9, 3263-3269. 
Shall, S. (1984). ADP-ribose in DNA repair: a new component of DNA excision repair Adv. Rad. Biol. 11, 1-69.

Simonin, F., Ménissier-de Murcia, J., Poch, O., Muller, S., Gradwohl, G., Molinete, M., Penning, C., Keith, G. and de Murcia, G. (1990). Expression and site directed mutagenesis of the catalytic domain of human poly(ADP-ribose) polymerase in Escherichia coli. J. Biol. Chem. 265, 19249-19256.

Simonin, F., Poch, O., Delarue, M. and de Murcia, G.

Identification of potential active-site residues in the human poly(ADP-ribose) polymerase.

(1993a) J. Biol. Chem. ( in press)

Simonin, F., Höfferer, L., Panzeter, P., Muller, S., de Murcia, G. and Althaus, F. The carboxy-terminal domain of human poly(ADP-ribose) polymerase: overproduction in Escherichia coli, large scale purification and characterization. (1993b) J. Biol. Chem. ( in press)

Molinete, M., Vermeulen, W., Bürkle, A., Ménissier-de Murcia, J., Küpper, J., Hoeijmakers, J. and de Murcia, G.

Overproduction of the poly(ADP-ribose) polymerase DNA-binding domain blocks alkylation-induced DNA repair synthesis in mammalian cells.

$E M B O J$. (submitted). 